(2) Open Access Full Text Article

\title{
Analysis of the Clinicopathological Characteristics of Stage I-III Colorectal Cancer Patients Deficient in Mismatch Repair Proteins
}

This article was published in the following Dove Press journal: OncoTargets and Therapy

\author{
Yichao Liang ${ }^{l, *}$ \\ Xinling Cai $\mathbb{D}^{2, *}$ \\ Xu Zheng ${ }^{2}$ \\ Hongzhuan Yin' \\ 'Department of General Surgery, \\ Shengjing Hospital of China Medical \\ University, Shenyang, Liaoning, People's \\ Republic of China; ${ }^{2}$ Department of \\ Clinical Oncology, Shengjing Hospital of \\ China Medical University, Shenyang, \\ Liaoning, People's Republic of China
}

*These authors contributed equally to this work
Correspondence: Hongzhuan Yin Department of General Surgery, Shengjing Hospital of China Medical University, Shenyang, Liaoning, People's Republic of China

Tel +8618940256969

Fax +86 2496615-10094

Email chad156@hotmail.com
Purpose: To investigate the clinicopathological characteristics of stage I-III colorectal cancer (CRC) patients with deficient mismatch repair (dMMR) protein.

Patients and Methods: A retrospective analysis of 61 patients with stage I-III CRC confirmed by immunohistochemistry as dMMR after radical resection at Shenjing Hospital of China Medical University from May 2017 to June 2019 was performed. A total of 183 stage I-III CRC patients with proficient mismatch repair (pMMR) protein from the same period were randomly selected as a control group. The clinicopathological data of the two groups were investigated.

Results: There were significant differences between the two groups in age, sex, site of onset, maximum diameter of tumor, T stage, tumor differentiation, and histological type $(P<0.05)$. No significant difference was detected in nerve vessel invasion, cancer nodules, the $\mathrm{N}$ stage or the TNM stage. In the dMMR group, 41 patients (66.13\%) showed PMS2/MLH1 deletion, and the number of MSH2/MSH6 deletion is 21 patients (33.87\%). Among them, 34 patients (54.84\%) had PMS2 and MLH1 deficiency. In total, 16 patients (25.81\%) had MSH2 and MSH6 deficiency. A total of 5 patients (8.06\%) showed simply PMS2 deletion and 5 patients $(8.06 \%)$ showed simply MSH6 deletion. In total, 2 patients $(3.23 \%)$ showed concurrent loss of PMS2, MLH1 and MSH2. No significant difference were found $(P>0.05)$ in the above factors among dMMR CRC patients with different MMR proteins deletions.

Conclusion: Our results show that dMMR status may be more likely exist in female and younger ( $\leq 55$ years) patients with a greater tumor burden $(>5 \mathrm{~cm})$, right colon, T4 stage disease, poor differentiation and mucinous adenocarcinoma. Loss of PMS2 and MLH1 is the most common pattern of MMR protein expression deficiency, followed by concurrent deletion of MSH2 and MSH6.

Keywords: deficient mismatch repair, clinicopathological characteristics, colorectal cancer, microsatellite instability

\section{Introduction}

Colorectal cancer $(\mathrm{CRC})$ is the third most common cancer in the world and has the second highest mortality rate. ${ }^{1}$ With economic development and changes in dietary habits, the incidence of $\mathrm{CRC}$ remains high. ${ }^{2} \mathrm{CRC}$ is divided into two types: sporadic CRC and genetic CRC. Abnormalities in multiple molecular pathways at the cellular level may lead to the occurrence of CRC. The main causes include chromosomal instability and microsatellite instability (MSI). ${ }^{3}$

Microsatellites are widely found in the genome and are prone to insertion or deletion mutations during DNA replication; the main function of the DNA 
mismatch repair (MMR) system is to maintain the stability of microsatellites. ${ }^{4}$ When the DNA MMR system is abnormal, errors in microsatellite sequences gradually accumulate during DNA replication, resulting in shortening or amplification of the corresponding sequences, producing MSI. ${ }^{5}$ Approximately $10-15 \%$ of CRC is caused by MSI. Hereditary CRCcaused by germline mutations in an MMR gene is rare; it is known as Lynch syndrome and accounts for approximately $2-5 \%$ of all CRCs. Most of the remaining CRCs are sporadic MSI CRC, which occurs on the basis of DNA MMR gene abnormalities, such as abnormal methylation of the MLH1 promoter CpG island, resulting in downstream gene inactivation. ${ }^{6}$

MSI is widely used in the clinical treatment of CRC and has important value in prognostic stratification, guiding adjuvant therapy, screening patients who may benefit from immunotherapy, and screening Lynch patients. Therefore, it is clinically necessary to screen CRC patients with MMR defects (ie, dMMR or MSI). Research on the expression of MMR in CRC and its relationship with clinicopathological characteristics is also particularly important.

Although genetic testing is the gold standard for detecting MSI, the College of American Pathologists (CAP) recommends screening for MSI by detecting the expression of MMR proteins. Studies have shown that the two methods are highly consistent, ${ }^{7}$ and immunohistochemistry is recommended as the screening method. Studies have shown that dMMR CRC has the following characteristics: the primary tumor is mostly located in the right colon, the tumor stage is earlier, Crohn's disease-like lymph node reaction is common, the tumor cells are poorly differentiated, most of them are rich in mucus and have signet ring-like changes, lymphocyte infiltration in the surrounding tissues is abundant, and BRAF activation mutations are common. ${ }^{8}$ However, there are relatively few studies on the correlation between MSI and cancer nodules, and there are controversial conclusions about the patients' tumor size, sex, age, vascular tumor thrombus and lymphatic metastasis. In this study, we aimed to assess the correlation between MMR status and the pathological features of CRC patients, to analyze the types of MMR protein expression loss, and to determine whether there are differences in the clinical pathological features among dMMR CRC patients with different types of MMR protein loss.

\section{Patients and Methods}

\section{Patients}

This retrospective cross-sectional study was performed in compliance with the Declaration of Helsinki. This study was approved by the IRC (Institutional Review Committee) of Shengjing Hospital of China Medical University. Due to the retrospective nature of this study, informed consents from patients were waived by the IRC. Patient data collected in this study were maintained with confidentiality.

The clinical and pathological data of patients who underwent radical surgery at Shengjing Hospital of China Medical University from May 2017 to June 2019 were collected; CRC was confirmed by pathology in each case (Shengjing Hospital of China Medical University now routinely tests MMR protein expression). dMMR CRC cases (ie, one or more of the 4 MMR proteins MLH1, MSH2, MSH6 and PMS2 were negative by immunohistochemistry) were selected. pMMR CRC cases (ie, all 4 proteins were positive by immunohistochemistry) were randomly selected as a control group during the same time period. The number of cases in the control group was 3 times that of the dMMR group.

The inclusion criteria were as follows: patients with (1) clear pathological diagnosis; (2) complete clinical data; and (3) pathological stage I-III.

The exclusion criteria were as follows: patients who (1) had incomplete clinical data; (2) received clinical trials related to treatment; (3) received adjuvant therapy before surgery; or (4) had synchronous or metachronous carcinoma. Finally, a total of $61 \mathrm{dMMR}$ patients and 183 pMMR patients were included in our study according to the inclusion and exclusion criteria.

The medical records, surgical records, pathological results and other clinical data of the selected patients were collected, and pathological staging was performed according to the 8th edition of the AJCC Colorectal Tumor Staging Manual. The patients' number of hospitalizations, sex, age, lesion location, maximum tumor diameter (pathological tissue), detailed pathological stage, tumor grade (degree of differentiation), histological type, neurovascular invasion status, cancer nodule status and MMR protein status were recorded.

\section{Statistical Analysis}

Measurement data that conformed to a normal distribution are presented as $\mathrm{x} \pm \mathrm{s}$, and those that did not conform are presented as the median (quartile interval). The $t$-test or 
Wilcoxon rank sum test was used to compare the differences between the means; the enumeration data were expressed as rates; $y^{2}$ test or Fisher's exact probability method was used to compare the differences between the rates. SPSS 23.0 was used for statistical analysis. A $P$ value $<0.05$ indicates statistical significance.

\section{Results}

\section{Differences in Clinicopathological Characteristics}

Age and sex: The average patient age in the pMMR group was $63.34 \pm 9.29$ years, and the patients in the dMMR group were slightly younger (60.07 \pm 13.17 years), but the difference was not statistically significant $(P>0.05$, Figure 1$)$. The subgroup analysis showed that the proportion of patients under 55 years old was $32.80 \%$ in the dMMR group, significantly higher than the $15.8 \%$ in the pMMR group, and the difference was statistically significant $(P<$ 0.01 , Table 1). The female proportion of the dMMR group was $54.1 \%$, higher than the $38.3 \%$ in the pMMR group, and the difference was statistically significant $(P<0.05$, Table 1). The ratio of men to women was $0.85: 1$ in the dMMR group, while that in the pMMR group was 1.61:1.

Location of primary tumor: Of all CRCs, $63.9 \%$ occurred in the right hemicolon in the dMMR group, which was significantly higher than the $18.6 \%$ occurrence in the pMMR group $(P<0.01$, Table 1$)$.

Maximum tumor diameter and $\mathrm{T}$ stage: The median diameter and quartile interval of the maximum tumor diameter were $6.0(4.0 ; 7.0) \mathrm{cm}$ in the dMMR group and $4.5(3.5 ; 5.5) \mathrm{cm}$ in the pMMR group, and the difference

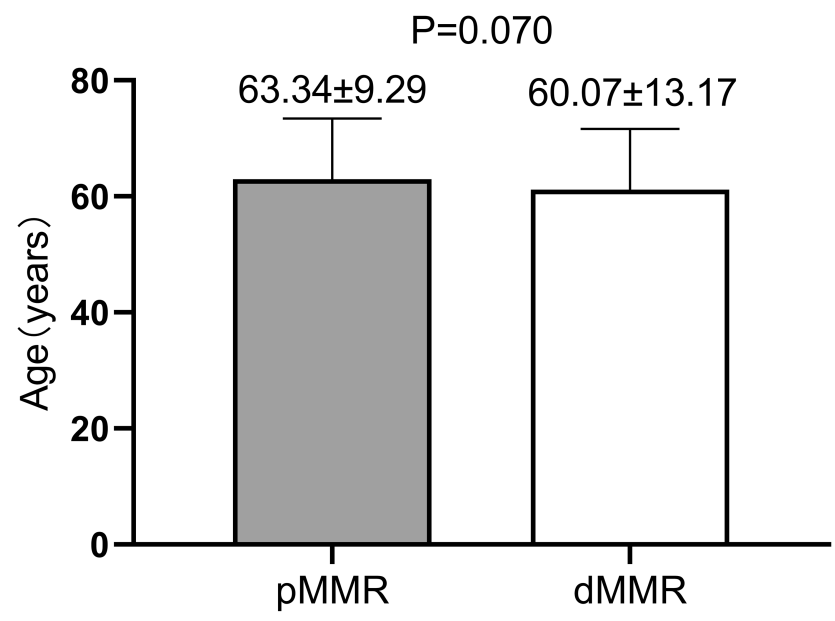

Figure I Age difference between the two groups. was statistically significant $(P<0.01$, Figure 2$)$. Subgroup analysis showed that $55.7 \%$ of the tumors in the dMMR group were larger than $5 \mathrm{~cm}$, significantly higher than the $28.4 \%$ in the pMMR group $(P<0.01$, Table 1$)$. Regarding the T stage, $62.3 \%$ of the patients in the dMMR group had T4 tumors, which was significantly higher than the $21.9 \%$ in the pMMR group $(P<0.01$, Table 1$)$.

TNM stage: In the dMMR group, the proportion of patients with pathological stage II was $58.1 \%$, which was significantly higher than that of other stages. Further analysis showed that the proportion of stage II patients in the dMMR group was slightly higher than that in the pMMR group (51.4\%), but there was no significant difference $(P>$ 0.05 , Table 1).

Differentiation degree and histological type: Patients with poorly differentiated tumors accounted for $41.0 \%$ of the dMMR group and $10.9 \%$ of the pMMR group, and the difference was statistically significant $(P<0.01$, Table 1$)$; however, moderately differentiated tumors accounted for the majority in both groups $(50.8 \%$ in the dMMR group and $82.5 \%$ in the pMMR group, see Table 1). Histologically, the proportion of mucinous adenocarcinomas was $19.7 \%$ in the dMMR group and $7.1 \%$ in the pMMR group, and the difference was statistically significant $(P<0.05$, Table 1$)$. In addition, there were 2 cases of medullary carcinoma in the dMMR group and 2 cases of undifferentiated adenocarcinoma and 1 case of signet-ring cell carcinoma in the pMMR group. The histological types of the two groups were mainly unspecified types of adenocarcinoma, with proportions of $77.0 \%$ and $91.8 \%$ (Table 1).

There was no significant difference between the two groups in terms of the presence of nerve vessel invasion, the presence of cancer nodules, or the $\mathrm{N}$ stage $(P>0.05$, Table 1).

\section{Characteristics of Colorectal Cancer with Missing Mismatch Protein Expression}

The clinicopathological data of dMMR CRC lacking different MMR proteins were analyzed, and it was found that the differences between the above factors were not statistically significant, and the $P$ values were all greater than 0.05 (Table 2). There was 1 patient who lacked the expression of the other three MMR proteins except MSH6. This patient was included in both the PMS2/MLH1 and MSH2/MSH6 subgroups in the statistical analysis. In the dMMR group, there 
Table I Comparison of Clinicopathological Data Between dMMR and pMMR Colorectal Cancer

\begin{tabular}{|c|c|c|c|c|c|c|}
\hline \multirow{2}{*}{ Age } & \multicolumn{2}{|c|}{ pMMR $n=183$} & \multicolumn{2}{|c|}{ dMMR $n=6 I$} & \multirow{4}{*}{$\begin{array}{l}\text { Total } \mathbf{n = 2 4 4} \\
195 \\
49\end{array}$} & \multirow{4}{*}{$\begin{array}{l}P \text { value } \\
0.004^{*}\end{array}$} \\
\hline & & & & & & \\
\hline$>55$ & 154 & $(84.2 \%)$ & 41 & $(67.2 \%)$ & & \\
\hline$\leq 55$ & 29 & $(15.8 \%)$ & 20 & $(32.8 \%)$ & & \\
\hline \multicolumn{7}{|l|}{ Sex } \\
\hline Male & 113 & $(61.7 \%)$ & 28 & (45.9\%) & $|4|$ & $0.030 *$ \\
\hline Female & 70 & $(38.3 \%)$ & 33 & (54.1\%) & 103 & \\
\hline \multicolumn{7}{|l|}{ Location } \\
\hline Left & 47 & $(25.7 \%)$ & 11 & (I8.0\%) & 58 & $0.000 *$ \\
\hline Right & 34 & (I8.6\%) & 39 & (63.9\%) & 73 & \\
\hline Rectum & 102 & $(55.7 \%)$ & II & (18.0\%) & 113 & \\
\hline \multicolumn{7}{|l|}{ Diameter } \\
\hline$\leqq 5 \mathrm{~cm}$ & $13 \mid$ & (71.6\%) & 27 & (44.3\%) & 158 & $0.000 *$ \\
\hline$>5 \mathrm{~cm}$ & 52 & $(28.4 \%)$ & 34 & (55.7\%) & 86 & \\
\hline \multicolumn{7}{|c|}{ Lymphovascular invasion } \\
\hline Absent & 109 & $(59.6 \%)$ & 42 & (68.9\%) & 151 & 0.196 \\
\hline Present & 74 & $(40.4 \%)$ & 19 & (31.I\%) & 93 & \\
\hline \multicolumn{7}{|c|}{ Extranodal extension } \\
\hline Absent & 160 & (87.4\%) & 57 & (93.4\%) & 217 & 0.195 \\
\hline Present & 23 & $(12.6 \%)$ & 4 & $(6.6 \%)$ & 27 & \\
\hline \multicolumn{7}{|l|}{ T stage } \\
\hline TI-3 & 143 & (78.1\%) & 23 & (37.7\%) & 166 & $0.000 *$ \\
\hline $\mathrm{T} 4$ & 40 & (21.9\%) & 38 & (62.3\%) & 78 & \\
\hline \multicolumn{7}{|l|}{$\mathrm{N}$ stage } \\
\hline No & 116 & (63.4\%) & 44 & (72.1\%) & 160 & 0.298 \\
\hline NI & 47 & $(25.7 \%)$ & 14 & $(23.0 \%)$ & 61 & \\
\hline N2 & 20 & $(10.9 \%)$ & 3 & $(4.9 \%)$ & 23 & \\
\hline \multicolumn{7}{|l|}{ TNM stage } \\
\hline I & 23 & $(12.6 \%)$ & 9 & (14.8\%) & 32 & 0.501 \\
\hline ॥ & 94 & (51.4\%) & 35 & (57.4\%) & 129 & \\
\hline III & 66 & $(36.1 \%)$ & 17 & $(27.9 \%)$ & 83 & \\
\hline \multicolumn{7}{|l|}{ Tumor grade } \\
\hline I & 12 & $(6.6 \%)$ & 5 & $(8.2 \%)$ & 17 & $0.000 *$ \\
\hline II & $|5|$ & $(82.5 \%)$ & 31 & (50.8\%) & 182 & \\
\hline III & 20 & $(10.9 \%)$ & 25 & (4I.0\%) & 45 & \\
\hline \multicolumn{7}{|l|}{ Tumor type } \\
\hline NOS & 168 & (91.8\%) & 47 & (77.0\%) & 215 & $0.003^{*},{ }^{a}$ \\
\hline Medullary & 0 & $(0.0 \%)$ & 2 & $(3.3 \%)$ & 2 & \\
\hline Undifferentiated & 1 & $(0.5 \%)$ & 0 & $(0.0 \%)$ & I & \\
\hline Signet ring & I & $(0.5 \%)$ & 0 & $(0.0 \%)$ & I & \\
\hline Mucinous & 13 & (7.1\%) & 12 & (19.7\%) & 25 & \\
\hline
\end{tabular}

Note: ${ }^{a}$ Fisher's exact test, ${ }^{*} \mathrm{P}<0.05$.

were 41 patients with PMS2/MLH1 deletions, accounting for $66.13 \%$ of cases, and 21 with MSH2/MSH6 deletions, accounting for $33.87 \%$ of cases (Figure 3). Among them, 5 cases were simply missing PMS2, accounting for $8.06 \%$ of cases; 16 cases were missing MSH2 and MSH6, accounting for $25.81 \%$ of cases; 5 cases were missing MSH6 alone, 
$P=0.000$

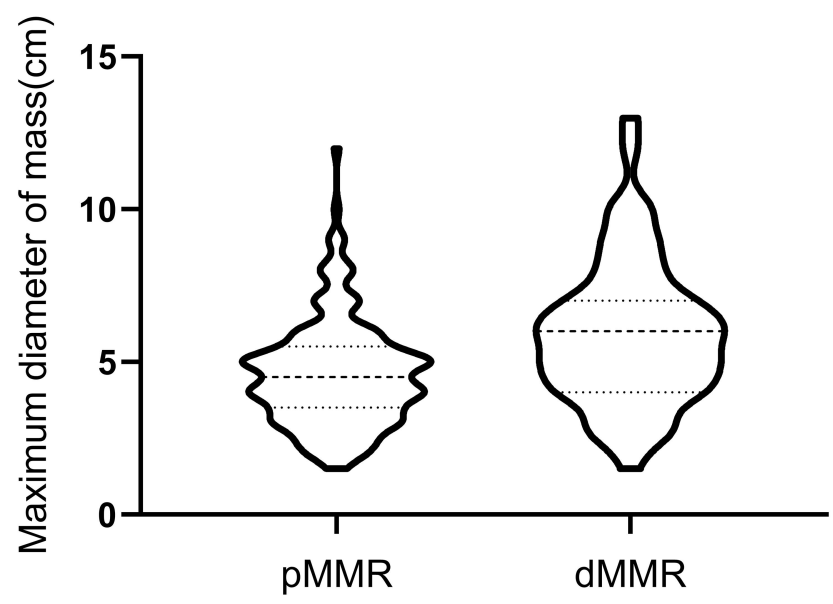

Figure 2 Difference in the maximum tumor diameter between the two groups.

accounting for $8.06 \%$ of cases; 34 cases were missing PMS2 and MLH1, accounting for $54.84 \%$ of cases; and only 2 cases were only positive MSH6 expression, accounting for 3.23\% of cases (see Figure 4). No patients were missing MLH1 expression alone, missing MSH2 expression alone, or missing all 4 MMR proteins.

\section{Discussion}

\section{Mismatch Repair System}

DNA MMR is one of the indirect repair mechanisms of DNA damage. Its function is to recognize and repair the loops generated on the double-stranded DNA due to mutations such as base mismatch, insertion or deletion, thereby maintaining the stability of the genome. ${ }^{9}$ When DNA damage occurs, an MSH heterodimer (MSH2-MSH6, also known as MutS $\alpha$; MSH2-MSH3, also known as MutS $\beta$ ) first binds to the mismatched DNA. MutS $\alpha$ recognizes the mismatched sequence of 1-2 base pairs; MutS $\beta$ recognizes the mismatched sequence of more than 2 base pairs and the insertion-deletion loop. ${ }^{10}$ After the mismatched DNA fragments are identified, MutS $\alpha / \mathrm{MutS} \beta$ recruits and binds to the MLH heterodimer (MLH1PMS2, also known as MutL $\alpha) .{ }^{11}$ When replication factor $\mathrm{C}$ (RFC) localizes proliferating cell nuclear antigen (PCNA), MutL $\alpha$ combines with proliferating cell nuclear antigen to enable PMS2 to exert endonuclease activity. ${ }^{12}$ With the participation of exonucleases, the wrong fragments are excised, but there are also cases that do not rely on exonucleases. ${ }^{13}$ Finally, the original DNA fragment that had the error is resynthesized with the help of DNA polymerase $\delta$ and DNA ligase. ${ }^{14}$ DNA MMR gene expression defects may lead to MSI, which is closely related to the occurrence and development of CRC. Sporadic MSI CRC is mostly caused by methylation of the MLH1 promoter. ${ }^{15}$ Although the gold standard for identifying MSI phenotypes is PCR amplification of microsatellite repeats, MSI patients can also be identified based on histopathology and immunohistochemistry results. The consistency between the two methods is high, and immunohistochemistry is more economically convenient. ${ }^{16}$ Immunohistochemistry was used to detect the expression of MMR proteins (MLH1, MSH2, MSH6, and PMS2) in CRC tissues. Patients with negative expression of 1 or more negative targets were suggested to be dMMR, ie, with microsatellite instability-high (MSI-H); those who had positive results for all 4 proteins were labeled as pMMR, ie, with microsatellite instability-low (MSI-L) or microsatellite stability (MS). At present, immunohistochemistry is widely used in screening MSI CRC. When screening for MSI-H CRC, care should be taken to exclude Lynch syndrome. BRAFV600E somatic mutations occur in approximately $40 \%$ of patients with sporadic MSI-H CRC but are very rare in Lynch syndrome. ${ }^{17}$ In addition to referring to the family history of the patient, the use of immunohistochemistry to determine the expression of the BRAF-V600E protein helps to exclude sporadic MSI CRC; ${ }^{18}$ it can be further combined with genetic testing to diagnose Lynch syndrome.

\section{Clinicopathological Characteristics of Patients with $\mathrm{dMMR}$}

Our research shows that stage I-III dMMR CRC has the following special clinicopathological characteristics: younger onset, more common in women, more common on the right side, greater tumor burden at diagnosis, more common in stage $\mathrm{T} 4$, lower differentiation degree, and a higher proportion of mucinous adenocarcinoma.

Multiple studies have confirmed that the onset age of dMMR CRC is younger than that of pMMR CRC. ${ }^{19}$ There is currently controversy as to whether there is a sex difference. Some scholars have found that it is more common in women,${ }^{20}$ which is consistent with our results, but there are also literatures showing that there is no significant difference between men and women. ${ }^{21}$ In addition, we found that the T stage of $\mathrm{dMMR} \mathrm{CRC}$ is late, and the degree of tumor invasion is deep, consistent with the conclusions of other scholars. ${ }^{22}$ Our research also showed that compared with patients with pMMR CRC, dMMR 
Table 2 Correlation Analysis of Mismatch Repair Protein Deletion Types and Clinicopathological Characteristics

\begin{tabular}{|c|c|c|c|c|}
\hline & PMS2/MLHI(-) n=4I & MSH2/MSH6 (-) n=2I & Total $n=62$ & $P$ value \\
\hline \multicolumn{5}{|l|}{ Age } \\
\hline$>55$ & $27(65.9 \%)$ & 15(7|.4\%) & 42 & 0.657 \\
\hline$\leqq 55$ & $14(34.1 \%)$ & $6(28.6 \%)$ & 20 & \\
\hline \multicolumn{5}{|l|}{ Gender } \\
\hline Male & $18(43.9 \%)$ & $10(47.6 \%)$ & 28 & 0.993 \\
\hline Female & $23(56.1 \%)$ & II (52.4\%) & 34 & \\
\hline \multicolumn{5}{|l|}{ Location } \\
\hline Left & $8(19.5 \%)$ & $4(19.0 \%)$ & 12 & $0.639^{\mathrm{a}}$ \\
\hline Right & $27(65.9 \%)$ & $12(57.1 \%)$ & 39 & \\
\hline Rectum & $6(14.6 \%)$ & $5(23.8 \%)$ & 11 & \\
\hline \multicolumn{5}{|l|}{ Diameter } \\
\hline$\leqq 5 \mathrm{~cm}$ & $19(46.3 \%)$ & $8(38.1 \%)$ & 27 & 0.535 \\
\hline$>5 \mathrm{~cm}$ & $22(53.7 \%)$ & $13(61.9 \%)$ & 35 & \\
\hline \multicolumn{5}{|c|}{ Lymphovascular invasion } \\
\hline Absent & $27(65.9 \%)$ & $16(76.2 \%)$ & 43 & 0.403 \\
\hline Present & $14(34.1 \%)$ & $5(23.8 \%)$ & 19 & \\
\hline \multicolumn{5}{|c|}{ Extranodal extension } \\
\hline Absent & $37(90.2 \%)$ & $20(95.2 \%)$ & 57 & 0.849 \\
\hline Present & $4(9.8 \%)$ & $\mathrm{I}(4.8 \%)$ & 5 & \\
\hline \multicolumn{5}{|l|}{ T stage } \\
\hline TI-3 & $16(39.0 \%)$ & $7(33.3 \%)$ & 23 & 0.872 \\
\hline $\mathrm{T} 4$ & $25(61.0 \%)$ & $14(66.7 \%)$ & 39 & \\
\hline \multicolumn{5}{|l|}{$\mathrm{N}$ stage } \\
\hline No & $27(65.9 \%)$ & $17(8 \mid .0 \%)$ & 44 & $0.584^{\mathrm{a}}$ \\
\hline $\mathrm{NI}$ & II (26.8\%) & $3(14.3 \%)$ & 14 & \\
\hline N2 & $3(7.3 \%)$ & $\mathrm{I}(4.8 \%)$ & 4 & \\
\hline \multicolumn{5}{|l|}{ TNM stage } \\
\hline I & $5(12.2 \%)$ & $4(19.0 \%)$ & 9 & $0.420^{\mathrm{a}}$ \\
\hline II & $22(53.7 \%)$ & $13(6 \mid .9 \%)$ & 35 & \\
\hline III & $14(34.1 \%)$ & $4(19.0 \%)$ & 18 & \\
\hline \multicolumn{5}{|l|}{ Tumor grade } \\
\hline I & $4(9.8 \%)$ & $\mathrm{I}(4.8 \%)$ & 5 & $0.925^{\mathrm{a}}$ \\
\hline II & $20(48.8 \%)$ & II (52.4\%) & 31 & \\
\hline III & $17(4 \mid .5 \%)$ & $9(42.9 \%)$ & 26 & \\
\hline \multicolumn{5}{|l|}{ Tumor type } \\
\hline NOS & $32(78.0 \%)$ & $\mid 5(7 \mid .4 \%)$ & 47 & $0.78 \mathrm{I}^{\mathrm{a}}$ \\
\hline Medullary & $2(4.9 \%)$ & I (4.8\%) & 3 & \\
\hline Mucinous & $7(17.1 \%)$ & $5(23.8 \%)$ & 12 & \\
\hline
\end{tabular}

Note: ${ }^{\text {a}}$ Fisher's exact test.

patients had a larger maximum tumor diameter and a higher percentage of tumors over $5 \mathrm{~cm}$. It suggests that the tumor volume of dMMR patients is larger at the time of diagnosis, which is consistent with the literature reports. ${ }^{20}$ In fact, in different stages of CRC, the incidence of MSI differs significantly. The incidence of dMMR in patients with stage II CRC is approximately $20 \%$; the incidence is $12 \%$ in patients at stage III and only $4 \%$ in patients at stage III $^{23}$ Although in our results there is no significant difference in tumor stage between dMMR and 


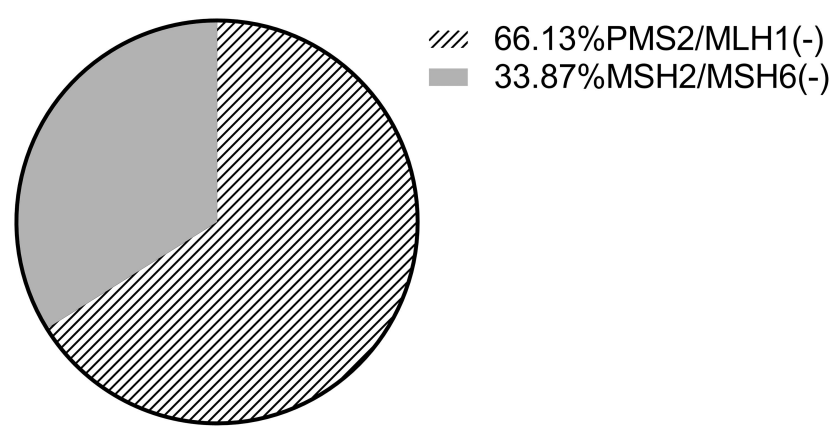

Figure 3 Proportion of different mismatch repair protein deletions.

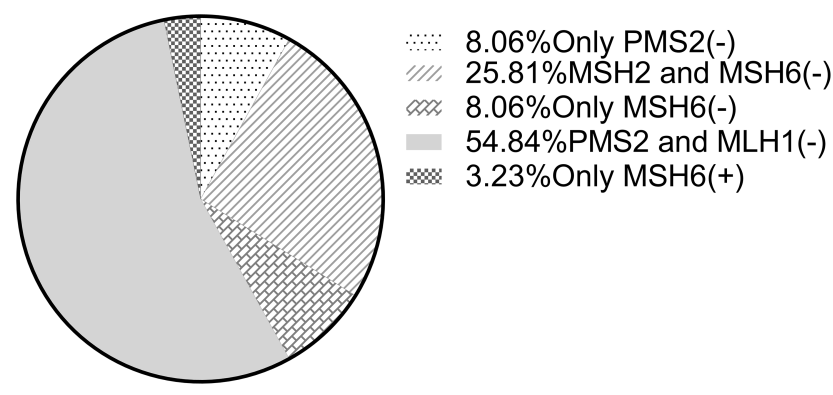

Figure 4 Hierarchical analysis of types of mismatch repair protein deletions.

pMMR patients, the proportion of stage II CRC is much higher than that of stage I and III CRC among dMMR patients. The neurovascular invasion, pathological stage, and $\mathrm{N}$ stage of $\mathrm{dMMR}$ patients were not different from those of pMMR patients, which was consistent with the published studies. $^{24}$

In terms of histological types, many studies have shown that there is a correlation between dMMR status and tumor histological type and degree of differentiation. In our study, dMMR patients had a higher proportion of colorectal mucinous adenocarcinoma and a lower degree of tumor differentiation, consistent with the study of Kaur et al. ${ }^{25}$ This suggests that the overall malignancy of patients with dMMR is higher. However, some studies have shown that in stage II patients, histological characteristics have no clear correlation with MSI. ${ }^{26}$ Further conclusions still need to be confirmed by large sample studies.

At present, there are few studies on MSI and cancer nodules. Our research found that the status of cancer nodules is not related to the loss of MMR protein expression. Cancer nodules are independent risk factors that indicate prognosis. ${ }^{27}$ However, there is currently controversy about the survival difference between N1b and N1c patients. There is no final conclusion about the effect of the number of cancer nodules on prognosis. Studies have shown that the count of CD8-positive T lymphocytes in cancer nodules of patients with CRC is significantly higher than that of tumor tissue, ${ }^{28}$ suggesting that changes in the tumor microenvironment are important for the generation of cancer nodules. The dMMR status has an important effect on the tumor microenvironment, ${ }^{29}$ suggesting that the generation of cancer nodules may be related to the microsatellite status. Our research did not draw a positive conclusion, which may be related to the stage of patients included and the small sample size. The specific relationship between the two and the mechanisms involved require more research and exploration.

\section{Type of Mismatch Repair Protein Expression Loss}

Among patients with negative MMR protein expression, we found that MLH1 and PMS2 coexpression loss was the most common, followed by concurrent deletion of MSH2 and MSH6. Among them, patients with PMS2 deletion alone accounted for only $8.06 \%$, and those with MSH6 deletion alone accounted for the same proportion. No separate deletion of MLH1 and MSH2 was found. It is suggested that the joint deletion of MLH1 and PMS2 as well as that of MSH2 and MSH6 are more common and that the single deletion is rare, consistent with the conclusions of other scholars. ${ }^{30}$ However, there are also studies showing that PMS2 deletion alone is not uncommon in dMMR patients, ${ }^{26}$ which may be related to the different stages of the patients included in the study. Further analysis found no differences in the clinical data of CRC patients with different types of MMR protein deletions, which is consistent with the results of Gandhi et al. ${ }^{26}$ This suggests that CRC patients with different types of dMMR proteins may have similar disease progression.

\section{Application of Microsatellite Instability in Colorectal Cancer}

Prompt effect on prognosis: Our results show that compared with pMMR CRC patients, dMMR patients have a younger onset age, more right side tumors, higher tumor burden, later T stage, lower differentiation degree and a higher proportion of mucinous adenocarcinoma. All of these factors are suggestive of poor prognosis. However, clinical practice and previous studies have shown that patients with early dMMR CRC have a relatively better prognosis than pMMR patients during the same period. ${ }^{31}$ In addition, studies have shown that the tumor site is an independent prognostic factor 
for $\mathrm{CRC}$; the prognosis of patients with right-sided colon cancer is significantly worse than that of patients with leftsided colon cancer and rectal cancer. ${ }^{32}$ Therefore, some scholars suggest that MSI should be screened for patients with right-sided colon cancer under the age of 60 to guide treatment and prognosis stratification. ${ }^{33}$ For patients with stage I-III CRC, MSI is a sign of good prognosis. ${ }^{34}$ The reason may be that MSI in patients with dMMR CRC leads to frameshift mutations, in turn producing a large number of related new antigens, which is more likely to trigger immune responses than patients with $\mathrm{pMMR},{ }^{29}$ and consequently increases the probability of tumor cells being recognized by the immune system. Although there are currently some patients with stage IV MSI-H CRC who may benefit from treatment with immune checkpoint inhibitors, MSI-H often indicates a poor prognosis in patients with metastatic stage IV CRC. ${ }^{35}$ This may be because the overexpression of immunosuppressive molecules counteracts the positive effect of lymphocyte infiltration. ${ }^{36}$ Further stratification studies by Goldstein et al found that BRAF-V600 mutations suggest poor prognosis in patients with stage IV microsatellite instable $\mathrm{CRC},{ }^{35}$ suggesting that these patients may have other molecular mechanism changes that affect prognosis. The specific related mechanism needs further exploration.

Guidance on treatment options: At present, domestic and foreign guidelines recommend adjuvant therapy for patients with CRC above stage II. The recurrence rate of patients with stage II MSI CRC is very low, so it is difficult to judge the effect of adjuvant therapy. Moreover, some scholars believe that patients with stage II and III MSI CRC cannot benefit from single-agent fluorouracil adjuvant chemotherapy. ${ }^{37}$ Whether it is necessary for stage II MSI CRC patients to receive adjuvant therapy requires more research evidence. Some researchers believe that these patients are more suitable for chemotherapy regimens containing oxaliplatin, ${ }^{38}$ which can be used as a reference in clinical practice. In recent years, the application value of immune checkpoint inhibitors in patients with metastatic advanced dMMR CRC has been confirmed. The overall response rate (ORR) of first-line immunotherapy in these patients is as high as $55 \%$. $^{39}$ This plays an important role in improving the survival of patients with stage IV MSI CRC.

\section{Conclusion}

Stage I-III MSI CRC has unique clinicopathological characteristics. Compared to MSS types, it generally occurs at a younger age and is more common in women. It is more likely to occur on the right side, the tumor load is higher, the $\mathrm{T}$ stage is later, the degree of differentiation is lower, and there is a higher proportion of mucinous adenocarcinoma. These features hint at the microsatellite status of patients with stage I-III CRC.

The status of cancer nodules in patients with stage I-III $\mathrm{CRC}$ is not related to the lack of MMR protein expression.

There is no difference in the clinicopathological characteristics of CRC patients with different types of MMR protein deletions in stages I-III, suggesting that CRC patients with different dMMR types may have a similar disease progression process. MSI is widely used in clinical practice. It is recommended that patients with stage II and III CRC be screened for MSI to guide the selection of treatment options and prognosis stratification.

To reveal the immune mechanism behind the special clinicopathological characteristics of MSI CRC patients and solve the immunotherapeutic problems encountered in treatment, it is necessary to further explore the relationship and interaction mechanism between MSI, infiltration of lymphocytes around the tumor and the body's immune response.

\section{Disclosure}

The authors report no conflicts of interest in this work.

\section{References}

1. Rawla P, Sunkara T, Barsouk A. Epidemiology of colorectal cancer: incidence, mortality, survival, and risk factors. Gastroenterol Rev. 2019;14(2):89-103. doi:10.5114/pg.2018.81072

2. Fatima $\mathrm{S}, \mathrm{Hu} \mathrm{X}$, Huang $\mathrm{C}$, et al. High-fat diet feeding and palmitic acid increase CRC growth in beta2AR-dependent manner. Cell Death Dis. 2019;10(10):711. doi:10.1038/s41419-019-1958-6

3. Penner-Goeke S, Lichtensztejn Z, Neufeld M, et al. The temporal dynamics of chromosome instability in ovarian cancer cell lines and primary patient samples. PLoS Genet. 2017;13(4):e1006707. doi:10.1371/journal.pgen.1006707

4. Vilar E, Gruber SB. Microsatellite instability in colorectal cancer-the stable evidence. Nat Rev Clin Oncol. 2010;7(3):153-162. doi:10.1038/ nrclinonc.2009.237

5. Kolodner RD, Marsischky GT. Eukaryotic DNA mismatch repair. Curr Opin Genet Dev. 1999;9(1):89-96. doi:10.1016/S0959-437X(99) 80013-6

6. Bedeir A, Krasinskas AM. Molecular diagnostics of colorectal cancer. Arch Pathol Lab Med. 2011;135(5):578-587. doi:10.5858/2010-0613RAIR.1

7. Hissong E, Crowe E, Yantiss R, et al. Assessing colorectal cancer mismatch repair status in the modern era: a survey of current practices and re-evaluation of the role of microsatellite instability testing. Mod Pathol. 2018;31(11):1756-1766. doi:10.1038/s41379-018-0094-7

8. Graham DM, Appelman HD. Crohn's-like lymphoid reaction and colorectal carcinoma: a potential histologic prognosticator. Mod Pathol. 1990;3(3):332-335. 
9. Michailidi C, Papavassiliou AG, Troungos C. DNA repair mechanisms in colorectal carcinogenesis. Curr Mol Med. 2012;12 (3):237-246. doi:10.2174/156652412799218859

10. Genschel J, Littman SJ, Drummond JT, et al. Isolation of MutSbeta from human cells and comparison of the mismatch repair specificities of MutSbeta and MutSalpha. J Biol Chem. 1998;273 (31):19895-19901. doi:10.1074/jbc.273.31.19895

11. Hombauer H, Campbell C, Smith C, et al. Visualization of eukaryotic DNA mismatch repair reveals distinct recognition and repair intermediates. Cell. 2011;147(5):1040-1053. doi:10.1016/j. cell.2011.10.025

12. Pluciennik A, Dzantiev L, Iyer R, et al. PCNA function in the activation and strand direction of MutL $\alpha$ endonuclease in mismatch repair. Proc Natl Acad Sci U S A. 2010;107(37):16066-16071. doi:10.1073/pnas.1010662107

13. Goellner EM, Putnam CD, Kolodner RD. Exonuclease 1-dependent and independent mismatch repair. DNA Repair (Amst). 2015;32:24-32. doi:10.1016/j.dnarep.2015.04.010

14. Longley M, Pierce A, Modrich P. DNA polymerase delta is required for human mismatch repair in vitro. J Biol Chem. 1997;272 (16):10917-10921. doi:10.1074/jbc.272.16.10917

15. Gupta S, Provenzale D, Llor X, et al. NCCN guidelines insights: genetic/Familial high-risk assessment: colorectal, version 2.2019. J Natl Compr Canc Netw. 2019;17(9):1032-1041. doi:10.6004/ jnccn.2019.0044

16. Li K, Luo H, Huang L, et al. Microsatellite instability: a review of what the oncologist should know. Cancer Cell Int. 2020;20(1):16. doi:10.1186/s12935-019-1091-8

17. McGivern A, Wynter CV, Whitehall VL, et al. Promoter hypermethylation frequency and BRAF mutations distinguish hereditary non-polyposis colon cancer from sporadic MSI-H colon cancer. Fam Cancer. 2004;3(2):101-107. doi:10.1023/B: FAME.0000039861.30651.c8

18. Anwar M, Murad F, Dawson E, et al. Immunohistochemistry as a reliable method for detection of BRAF-V600E mutation in melanoma: a systematic review and meta-analysis of current published literature. J Surg Res. 2016;203(2):407-415. doi:10.1016/j. jss.2016.04.029

19. Kim Y, Min B, Kim S, et al. Difference between proximal and distal microsatellite-unstable sporadic colorectal cancers: analysis of clinicopathological and molecular features and prognoses. Ann Surg Oncol. 2010;17(5):1435-1441. doi:10.1245/s10434-009-0888-4

20. Batur S, Vuralli Bakkaloglu D, Kepil N, et al. Microsatellite instability and B-type Raf proto-oncogene mutation in colorectal cancer: clinicopathological characteristics and effects on survival. Bosn J Basic Med Sci. 2016;16 (4):254-260. doi:10.17305/bjbms.2016.1238

21. Li W, Li H, Liu R, et al. Comprehensive analysis of the relationship between RAS and RAF mutations and MSI status of colorectal cancer in Northeastern China. Cell Physiol Biochem. 2018;50(4):1496-1509. doi:10.1159/000494649

22. Kang S, Na Y, Joung S, et al. The significance of microsatellite instability in colorectal cancer after controlling for clinicopathological factors. Medicine (Baltimore). 2018;97(9):e0019. doi:10.1097/ MD.0000000000010019

23. Ciardiello D, Vitiello PP, Cardone C, et al. Immunotherapy of colorectal cancer: challenges for therapeutic efficacy. Cancer Treat Rev. 2019;76:22-32. doi:10.1016/j.ctrv.2019.04.003

24. Ismael N, El Sheikh S, Talaat S, et al. Mismatch repair proteins and microsatellite instability in colorectal carcinoma (MLH1, MSH2, MSH6 and PMS2): histopathological and immunohistochemical study. Open Access Maced J Med Sci. 2017;5(1):9-13. doi:10.3889/ oamjms.2017.003
25. Kaur G, Masoud A, Raihan N, et al. Mismatch repair genes expression defects \& association with clinicopathological characteristics in colorectal carcinoma. Indian J Med Res. 2011;134:186-192.

26. Gandhi J, Goswami M, Sharma A, et al. Clinical impact of mismatch repair protein testing on outcome of early staged colorectal carcinomas. J Gastrointest Cancer. 2018;49(4):406-414. doi:10.1007/s12029-017-9954-5

27. Kim C, Kim J, Park Y, et al. Prognostic implications of extranodal extension in relation to colorectal cancer location. Cancer Res Treat. 2019;51(3):1135-1143. doi:10.4143/crt.2018.392

28. Fassan M, Vianello L, Sacchi D, et al. Assessment of intratumor immune-microenvironment in colorectal cancers with extranodal extension of nodal metastases. Cancer Cell Int. 2018;18(1):131. doi:10.1186/s12935-018-0634-8

29. Guidoboni M, Gafà R, Viel A, et al. Microsatellite instability and high content of activated cytotoxic lymphocytes identify colon cancer patients with a favorable prognosis. Am J Pathol. 2001;159 (1):297-304. doi:10.1016/S0002-9440(10)61695-1

30. Yuan L, Chi Y, Chen W, et al. Immunohistochemistry and microsatellite instability analysis in molecular subtyping of colorectal carcinoma based on mismatch repair competency. Int J Clin Exp Med. 2015;8(11):20988-21000.

31. Sinicrope FA, Foster NR, Thibodeau SN, et al. DNA mismatch repair status and colon cancer recurrence and survival in clinical trials of 5-fluorouracil-based adjuvant therapy. J Natl Cancer Inst. 2011;103 (11):863-875. doi:10.1093/jnci/djr153

32. Nakagawa-Senda H, Hori M, Matsuda T, et al. Prognostic impact of tumor location in colon cancer: the Monitoring of Cancer Incidence in Japan (MCIJ) project. BMC Cancer. 2019;19(1):431. doi:10.1186/ s12885-019-5644-y

33. Chou C, Lin J, Wang H, et al. Microsatellite instability screening should be done for right-sided colon cancer patients less than 60 years of age. Int J Colorectal Dis. 2010;25(1):47-52. doi:10.1007/ s00384-009-0815-y

34. Kim C, Ahn J, Jung M, et al. Effects of microsatellite instability on recurrence patterns and outcomes in colorectal cancers. Br J Cancer. 2016;115(1):25-33. doi:10.1038/bjc.2016.161

35. Goldstein J, Tran B, Ensor J, et al. Multicenter retrospective analysis of metastatic colorectal cancer (CRC) with high-level microsatellite instability (MSI-H). Ann Oncol. 2014;25(5):1032-1038. doi:10.1093/ annonc/mdu 100

36. Marisa L, Svrcek M, Collura A, et al. The balance between cytotoxic t-cell lymphocytes and immune checkpoint expression in the prognosis of colon tumors. J Natl Cancer Inst. 2018;110(1):110. doi:10.1093/jnci/djx136

37. Sargent D, Marsoni S, Monges G, et al. Defective mismatch repair as a predictive marker for lack of efficacy of fluorouracil-based adjuvant therapy in colon cancer. J Clin Oncol. 2010;28(20):3219-3226. doi:10.1200/JCO.2009.27.1825

38. André T, de Gramont A, Vernerey D, et al. Adjuvant fluorouracil, leucovorin, and oxaliplatin in stage II to III colon cancer: updated 10-year survival and outcomes According to BRAF Mutation and Mismatch Repair Status of the MOSAIC Study. J Clin Oncol. 2015;33(35):4176-4187. doi:10.1200/JCO.2015.63.4238

39. Overman M, Lonardi S, Wong K, et al. Durable clinical benefit with nivolumab plus Ipilimumab in DNA mismatch repair-deficient/microsatellite instability-high metastatic colorectal cancer. J Clin Oncol. 2018;36(8):773-779. doi:10.1200/JCO.2017.76.9901 


\section{Publish your work in this journal}

OncoTargets and Therapy is an international, peer-reviewed, open access journal focusing on the pathological basis of all cancers, potential targets for therapy and treatment protocols employed to improve the management of cancer patients. The journal also focuses on the impact of management programs and new therapeutic agents and protocols on patient perspectives such as quality of life, adherence and satisfaction. The manuscript management system is completely online and includes a very quick and fair peer-review system, which is all easy to use. Visit http://www.dovepress.com/ testimonials.php to read real quotes from published authors.

Submit your manuscript here: https://www.dovepress.com/oncotargets-and-therapy-journal 